\title{
VLA OBSERVATIONS OF AN OPTICALLY DEEP SAMPLE OF MOLONGLO QUASARS: ASPECT DEPENDENCE OF THE OPTICAL CONTINUUM
}

\author{
V.K.Kapahi ${ }^{1,2}$, C.R.Subrahmanya ${ }^{2}$ and S.D'Silva ${ }^{2}$ \\ 1 JPL-Caltech, 238-700, Pasadena, Ca 91109, U.S.A. \\ 2 TIFR Centre, P.B.No.1234, Bangalore 560012, India
}

Quasars found in low frequency radio surveys are often assumed to have their jet axes randomly oriented in the sky because relativistically beamed radio cores rarely contribute significantly to the total flux density at metrewaves. But an orientation bias can still arise from the optical magnitude limit of the sample (Kapahi \& Shastri 1987) if radio beaming is accompanied by an enhancement of the optical continuum as well (due to beaming or other effects). Such a bias can explain the finding (de Ruiter et al. 1986) that the typical value of $R$ (ratio of core to extended flux density at $5 \mathrm{GHz}$ ) for the magnitude limited Bologna sample is about 5 times larger than for 3CR quasars, eventhough both samples are from low frequency surveys. It is thus consistent with the unified scheme for quasars (Orr \& Browne 1982; Kapahi \& Saikia 1982), which in fact requires an aspect dependence of the optical continuum (eg. Browne \& Wright 1985; Browne \& Murphy 1987).

In the Bologna sample, while the magnitude distribution for the high-R objects peaks well above the PSS plate limit, that for the low-R sources peaks at or below the plate limit, suggesting a change of 2 to $3 \mathrm{mag}$ due to orientation. For consistency, the unified scheme requires that most of the Bologna sources that turn out to be quasars from deeper optical identifications should have relatively weak radio cores. We report here a prreliminary finding relevant to this prediction from a project that we have recently undertaken to study the radio morphology of a new sample of southern quasars selected at $408 \mathrm{MHz}$ and based on identifications to a considerably fainter level.

\section{SAMPLE DEFINITION AND OBSERVATIONS}

Our new quasar sample has been derived from observations with the Molonglo Synthesis Telescope at $843 \mathrm{MHz}$ of about 700 sources from the $408 \mathrm{MHz}$ Molonglo Reference Catalogue (Large et al. 1981). All MRC sources with $S_{408} \geq 0.95 \mathrm{Jy}$ in the declination strip $-30^{\circ}<\delta<-20^{\circ},|b|>20^{\circ}$, were observed at several hour angles in the 'short cut' mode (Subrahmanya \& Hunstead 1986; Subrahmanya, in preperation). The fan beam images were CLEANed and restored with 30 arcsec Gaussian beams. The resulting positional accuracy is generally about 1 " in RA and 2" in DEC, sufficient to make highly reliable identifications from positional coincidence alone.

Identifications were made (Subrahmanya \& D'Silva, in preperation) using the SRC-J plates with a limiting blue magnitude of $\approx 23$. All 'stellar' identifications were taken to be quasars. On the SRC plates the stellar/fuzzy nature can be ascertained quite reliably down to atleast $\approx 22$ mag. It is unlikely that any quasar down to $\approx \mathbf{2 2 . 5} \mathrm{mag}$ would have been missed in this procedure. Only about $15 \%$ of the candidates were, however, fainter than $21 \mathrm{mag}$ (Fig.1), which is not surprising in view of the relatively high limiting flux density of the sample.

A subset of 75 sources, not previously mapped with high angular resolution, were observed by us recently using the VLA at $5 \mathrm{GHz}$ in the A/B hybrid configuration in the snapshot mode. After calibration, the data were reduced on the AIPS system at the VLA site. The rms noise on the maps was typically $\approx 0.1-0.2 \mathrm{mJy}$ and the angular resolution about $0.7-1.5$ arcsec.

\section{RESULTS AND DISCUSSION}

The distributions of the obseved values of $\mathrm{R}$ for the brighter $\left(\mathrm{m}_{b}<19.5\right)$ and fainter $\left(\mathrm{m}_{b} \geq 19.5\right)$ objects are shown in Fig.2. Not included in the Fig. are about 15 quasars of the steep-spectrum 
compact radio structure (mostly in the optically fainter group) and another 10 or so with uncertin radio core identification. The distributions appear to differ significantly from that for the $3 \mathrm{CR}$ quasars (assumed to be randomly oriented) in two respects; (i) strongly core-dominted $(R>10)$ sources appear to be more common, particularly in the optically brighter group, and (ii) the peak of the R-distribution for the brighter quasars appears to have moved to larger values of $R$ compared to the 3CR sample, while that for the fainter quasars seems to shift to lower values of $R$.

It is interesting that in both the above respects the observations are qualitatively in agreement with predictions of the unified scheme when the aspect dependence of the optical continuum is taken into account. Two obvious causes for such a dependence are relativistic beaming and geometrically thin (but optically thick) accretion disks (considered responsible for the 'blue bump'). Their angular dependences are, however, very different; while beaming dominates at small angles to the line of sight, the most rapid change in projected area of a disk occurs at large angles. It is therefore expected that limiting a sample by optical magnitude would influence large values of $R$ for optical beaming and relatively smaller values of $R$ for thin disks. The observed differences at low as well as high values of $R$ then imply that both the effects perhaps contribute to the blue continuum.

It is of course possible to think of other explanations, like an intrinsic correlation between optical and radio core luminosities. Optical spectroscopic observations of the complete sample would be extremely useful in further investigating the aspect dependence.

If the optical continuum does indeed depend on orientation as suggested by the observations reported here, then samples of radio quasars that are magnitude limited or based on a uv excess (true of almost all existing samples) are likely to be biased with regard to their jet axes and should be used with caution in investigating many of their properties that may depend on orientation.

VKK wishes to thank the Director, NRAO, for a visiting appointment during which the VLA observations and data reduction were carried out.

\section{REFERENCES}

Browne,I.W.A. \& Wright,A.E. 1985, MNRAS, $213,97$.

Browne,I,W.A. \& Murphy,D.W. 1987, MNRAS, 226, 601.

de Ruiter,H.R.,Rogora,A. \& Padrielli,L. 1986, in 'Quasars',IAU Symp.119,eds. G.Swarup \&

V.K.Kapahi, p197( D.Reidel, Dordrecht).

Kapahi,V.K. \& Saikia,D.J. 1982, J.Astrophys. Astron., 3, 465.

Kapahi,V.K. \& Shastri,P. 1987, MNRAS, 224, 17p.

Large,M.I.,Mills,B.Y.,Little,A.G.,Crawford,D.F. \& Sutton,J.M. 1981, MNRAS, 194, 693.

Orr,M.J.L. \& Browne,I.W.A. 1982, MNRAS, 200, 1067.

Subrahmanya,C.R. \& Hunstead,R.W. 1986, A\&A, 170, 27.

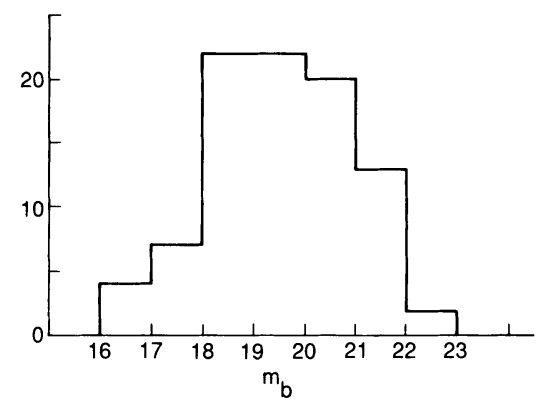

Fig.1. Magnitude distribution of quasar identifications in the Molonglo $1 \mathrm{Jy}$ sample.
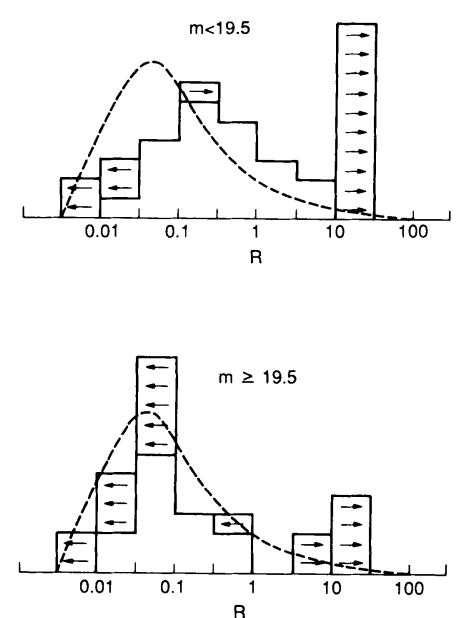

Fig.2. R-distributions for the brighter(top) and fainter(bottom) quasar identifications. Arrows indicate upper and lower limits. Curve shows smoothed distribution for all 3CR quasars from Orr \& Browne (1982). 